\title{
Hydraulic Fracture Orientation and Stress Field Revealed by Surface Based Microseismic Monitoring
}

\section{A Case Study of Fracturing Tight Sands Reservoir in Ordos Basin, China}

\author{
Zhengguang Zhao \\ Beijing GeoSun Energy Tech., Ltd \\ Beijing, China \\ zhzhg.uq@gmail.com
}

\author{
Ruizhao Yang \\ College of Geoscience \& Surveying Engineering \\ China University of Mining \& Technology at Beijing \\ Beijing, China \\ yrz@cumtb.edu.cn
}

\begin{abstract}
Surface based micro-seismic monitoring of well stimulation by hydraulic fracture now has been accepted by domestic oil and gas industry. Micro-seismic data can be used to evaluate the stimulation result, predict reservoir stress state and guide the drilling of horizontal laterals, etc. Surface based microseismic monitoring methods and the corresponding data processing technique naming vector scanning of micro-fracture has successfully mapped the hydraulic fractures. Through analysis of the spatial and temporal distribution of fracture energy, the hydraulic fracture process is understood. The current stress state of the stimulated reservoir is then determined by integrating the available geologic information and interpreted micro-seismic data.
\end{abstract}

Keywords-hydraulic fracture; sterss field; microseismic monitoring; semblance

\section{INTRODUCTION}

We present a workflow of surface based micro-seismic monitoring of a hydraulic fracture well stimulation in tight sands, Ordos Basin, China. The widely applied star pattern surface array may consists of more than 1000 channels with $6000-24000$ geophones stretching $2-10 \mathrm{~km}$ across and require several days for a 20-40-person crew to deploy the array. Considering the huge cost of the dense array of geophones, a rather sparser array of 3-component geophone is used in order to lower the monitoring cost while mapping the stimulated fracture network effectively. The micro-seismic location technique matching the sparser distribution of geophones, which is referred to as Vector Scanning Technique for Microfractures, is modified semblance methods based upon stacking waves without arrival-time picking ${ }^{[1]}$. Released fracture energy are scanned out and interpreted to represent the hypocenters of discrete micro-seismicity induced by hydraulic fracturing ${ }^{[2,3]}$. This study aims to present the methodology that how to effectively use micro-seismic data to help understand the stress field and rock failure mechanism.

The target perforation interval is a low permeability finegrained sand reservoir that requires fracture stimulation to facilitate oil production. WB4 well is a vertical well penetrating into the reservoir and is completed with a hydraulic fracture treatment. Hydraulic fractures are anticipated to propagate in the direction of the prevailing maximum horizontal compressive stress $(\sigma \mathrm{H})$. The mapped fracture geometry by surface based micro-seismic monitoring is also expected to be able to help understand the current stress state and find whether the natural fractures exist or not. These information is important to hydraulic fracture treatment optimization, fracture model calibration, well placement and reservoir production performance improvement and will provide robust guidance for drilling horizontal wells.

\section{Geological Setting}

WB4 well is a vertical exploration well drilled in North China Oil Field which is located in southern Ordos Basin, China. The perforated interval (1029.5 1035.0 m) is in Chang 7 Formation, of which the thickness of the sandbody is $12.1 \mathrm{~m}$. It is convenient for implementing hydraulic fracture stimulation because the overlying and underlying formation are thick mudstones.

The direction of SH is indicated by a series of near-well folds and small faults. The trend of these faults are in the range of $\mathrm{NW} 20^{\circ} \sim \mathrm{NW} 60^{\circ}$. The axes of these surrounding folds are in the direction of $\mathrm{NE} 20^{\circ} \sim \mathrm{NE} 70^{\circ}$, indicating the maximum horizontal compressive stress is in the direction of $\mathrm{NW}_{2}^{\circ} \sim$ $\mathrm{NW} 60^{\circ}$ along with those faults trend .

The objective of fracture and formation testing was to further understand the oil-bearing property and production performance of stimulated Chang 7 Formation. The simultaneous micro-seismic monitoring aimed to understand the hydraulic fracture geometry, current stress state and to diagnose the stimulation performance.

\section{DATA ACQUISITION}

The surface geophone array consisted of 20, 3-component stations arranged in concentric circles centered on the WB4 
wellhead. The nearest geophone to the well site is more than $1 \mathrm{~km}$ in order to keep away from the noise of truck engine, pump and electric generator. The farthest geophone is at a distance no more than $3 \mathrm{~km}$ to the well site for that the microseismic event signal will attenuate too much traveling a distance longer than it.

The micro-seismic data were collected using surface buried ( $<1 \mathrm{~m}$ in depth) 3-component geophone tools. The tools were equipped with 3 vertical sensors of $4.5 \mathrm{~Hz}$ in series and 3 horizontal sensors of $8 \mathrm{~Hz}$ in series. All geophone were oriented to east for calibration during data processing. Data were continuously recorded and sampled at $1 \mathrm{~ms}$ during 2.5 hour period among which the stimulation time is 115 minutes. Abutting 2 hour records were written to a memory card instrumented on each of the geophone tool. The array was live before during and after the pumping operation so that the background noise level can be recorded and analyzed.

\section{LOCATION METHOD}

At first, the recorded micro-seismic data were displayed in a manner of simultaneously showing separated vertical component trace and horizontal traces to check if the geophone had been properly working during hydraulic fracture. After this check, the effective recorded data were bandpass filtered to 1$45 \mathrm{~Hz}$ and then balanced using a trace by trace AGC.

At second, the micro-seismic data were split into a SEGY file containing 2.5 minutes recordings. Based on the waveform variance of noise and signal, the very obvious noise trace interval were picked out and removed. Only edited traces were delivered to vector scanning.

At third, we constructed a layered, 3D P-wave velocity model. This velocity model was interpolated within 3D grid space using an input $1 \mathrm{D}$ velocity model which was created by use of the available sonic log of WB4 well. The upper portions of the velocity model were extrapolated using expected rock properties. As the area where WB4 well was drilled is covered by hundreds of meters of loess and has a complex, rise and fall topography, the velocity model was calibrated carefully by integrating sonic logs and geologic reports of adjacent wells. After creating 3D velocity models, travel-time difference (i.e. the moveout across the array) table will be calculated based on the established velocity models.

Finally, semblance weighted stacking values, which are defined as "energies", of a series of records were calculated to examine the spatial and temporal distribution of acoustic emission energy ${ }^{[4]}$. The energy for each cell in a 3D grid was calculated for a series of time periods of one second up to a minute. Horizontal and vertical component beam sum records were analyzed for areas of concentrated energy. The highest energy cells for each time period were plotted and animated to show the relationship between the onset of the activity and the surface pumping pressure.

\section{RESULTS AND DISCUSSION}

A series of depth slice showing the fracture energy distribution in space within the specific time interval $(2.5$ minutes) were produced, thus the spatial and temporal distribution of hydraulic fracture energy can be examined and analyzed.

The vector scanning output shows that seismic activities had begun as the slurry rate initially increased. Within the preliminary 50 minutes of the stimulation, the released fracture energy is rather low, indicating fewer seismicity had been induced and no major hydraulic fracture had been created. This phenomenon also implies that natural fractures do exist in the tight sand reservoir, which is consistent with the local tectonic activity as indicated by many folds around the well site.

Two energy spikes occurred later, one is at around 60 minutes and the other 110minutes after the stimulation had begun. At these times, the slurry rate had a sudden decrease, indicating major hydraulic fractures were created.

By summing all the micro-seismic energy of specific time interval (2.5 minutes) within the stimulation operation, an energy summation depth slice at $1035 \mathrm{~m}$ from surface was calculated over the entire 2.5 hour period.

While a short 2.5-minute period containing the highest seismic energy can be used to determine at what time the major hydraulic fractures were created and in which direction they had propagated, the energy summation depth slice at specific depth can be used to study the hydraulic fracture geometry and the current stress state.

Shawn Maxwell et al. concluded that typically hydraulic fractures are considered to be single tensile fractures created orthogonal to the direction of minimum principal stress ${ }^{[5,6]}$. However, the injected fluid will follow a path of least resistance and depending on the stress conditions, may intersect pre-existing planes of weakness associated with either healed or open fractures. The resulting hydraulic fracture is therefore more complex than a single hydraulic fracture plane, consisting of an entire fracture network. Maxwell's view can be applied to our case and help to interpret the fracture process.

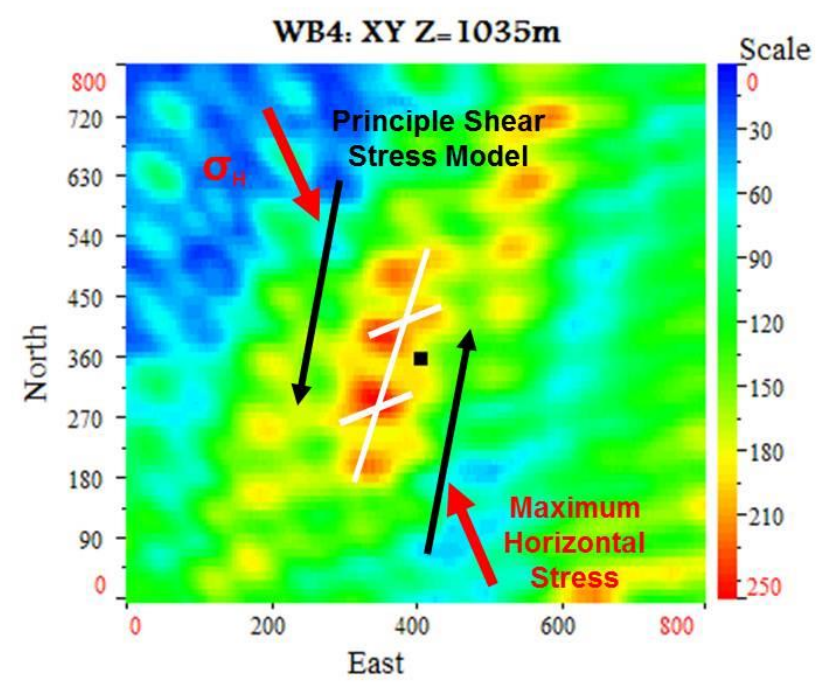

Fig.1. Interpreted shear fracture mechanism of the hydraulic fracture propagation within the reservoir. 
Preliminarily, the major hydraulic fractures were anticipated to propagate in the direction of the prevailing maximum horizontal compressive stress $\left(\sigma_{H}\right)$, which is in the direction of NW. However, Fig.1 shows that the major hydraulic fractures had propagated in the direction of $\mathrm{NE} 15^{\circ}$.

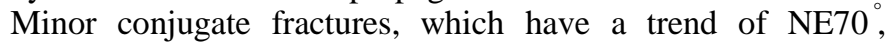
connected with the major fractures. These two sets of hydraulic fractures of different directions formed a complex fracture network and indicated the principle horizontal stress is in the direction of NE.

The discrepancy of the anticipated fracture propagation with the realistic fracture propagation may at first let us draw a conclusion that one of them must be wrong. But when noting the energy contour has a relatively larger area, this conclusion may be changed to another. Essentially, the image conveys a sense of the uncertainty in event location. The peaks of the energy contours are the most likely location of the event. The relatively larger peak area indicate high level of energy released by seismic activities, this can be interpreted as the initial hydraulic fracture had endured a dilation process. At this point, a shear fracture model (Fig.1) was introduced to help interpret the discrepancy between realistic and predicted stress orientation. Then one reasonable interpretation may be that the maximum horizontal stress $\left(\sigma_{\mathrm{H}}\right)$ of NW $25^{\circ}$ had formed shear $\operatorname{stress}\left(\tau_{\max }\right)$ which was at an angle to $\sigma_{\mathrm{H}}$, the propagation of the hydraulic fractures was then controlled by the shear stress. The shear fracture process was accompanied with hydraulic fracture dilation, resulting in a relatively larger peak energy area extent.

\section{CONCLUSIONS}

Surface based micro-seismic monitoring with vector scanning technique has successfully mapped the hydraulic fractures induced by the 115-minute hydraulic fracture operation of WB4 well. From the analysis of the spatial and temporal distribution of fracture energy, it can be determined that the major fracture process was occurred at around 60 minutes and 110 minutes after the stimulation begun. By integrated interpretation of the energy summation depth slice at $1035 \mathrm{~m}$, the current stress state was verified as the same with the predicted stress orientation before the stimulation and a shear fracture process was found, leading to a better understanding of the reservoir property and its stress field.

\section{ACKNOWLEDGMENT}

While we are not allowed to mention our client referred in this paper by name, we are grateful to them for permission to present some of the resulting figures and publish the results of the study referred in this paper. We also thank Beiyuan Liang for having provided necessary technical guidance and support for this study.

\section{REFERENCES}

[1] C. Shen, B.Y. Liang and Z.T. Li, "Principle of vector scanning technique for micro-fractures," ACTA PETROL EI SINICA, 2009, 30(5), pp.744-748.

[2] J.D. Lakings, P.M. Duncan, C. Neale and T. Theiner, "Surface based microseismic monitoring of a hydraulic fracture well stimulation in the Barnett Shale," 76th Annual International Meeting, 2006, SEG Expanded Abstracts, pp.605-608.

[3] P.M. Duncan, J.D. Lakings and R.A. Flores, "Method for passive seismic emission tomography," U. S. Patent Application Publication US 2008/0068928A1

[4] R.Z Yang, Z.G. Zhao, W.J. Peng, Y.B. Gu, Z.G. Wang and X.Q. Zhuang, "Integrated application of 3D seismic and microseismic data in the development of tight gas reservoirs," Applied Geophysics, 2013, 10(2): pp.157-169.

[5] S.C. Maxwell, D. Cho and M. Norton, "Integration of surface seismic and microseismic part 2: Understanding hydraulic fracture variability through geomechanical integration," CSEG RECORDER, 2011, 29, pp.26-30.

[6] S.C. Maxwell, D. Cho, T. Pope, et al., "Enhanced reservoir characterization using hydraulic fracture microseismicity," SPE140449. 